\title{
NONLINEAR BUCKLING FORMULATIONS AND IMPERFECTION MODELS FOR SHEAR DEFORMABLE PLATES BY THE BOUNDARY ELEMENT METHOD
}

\author{
Judha Purbolaksono And M. H. (FERri) Aliabadi
}

\begin{abstract}
This paper presents a nonlinear buckling analysis of shear deformable plates. Two models of imperfections are introduced: small uniform transverse loads and distributed transverse loads, according to the number of half-waves indicated by the eigenvectors from linear elastic buckling analysis. A simple numerical algorithm is presented to analyze the problems. Numerical examples with different geometries, loading and boundary conditions are presented to demonstrate the accuracy of the formulation.
\end{abstract}

\section{Introduction}

Plate buckling behavior has been investigated analytically and experimentally since the first experimental observation, almost 150 years ago; see [Walker 1984] for a review. Analytical solutions of linear buckling of plates based on classical plate theory can be found in [Brush and Almroth 1975; Timoshenko and Gere 1961]. Numerical methods have also been used [Bao et al. 1997; Liu 2001; Manolis et al. 1986; Purbolaksono and Aliabadi 2005b]. Liu [1987] and Syngellakis [1998] applied the boundary element method (BEM) to the stability analysis of thin plates. In [Purbolaksono and Aliabadi 2005a] we developed a boundary element method for analyzing linear buckling problems of shear deformable plates.

The boundary element method has also been applied to the analysis of nonlinear plate problems. Early works on geometrically nonlinear shear deformable plates by boundary element method include [Lei et al. 1990; He and Qin 1993], while Marczak and de Barcellos [1998] reported on a nonlinear stability analysis in shear deformable plates by the BEM. Other works contributing to BEM analysis of nonlinear buckling of thin plates have been made [Kamiya et al. 1984; Qin and Huang 1990; Tanaka et al. 1999].

Here we perform a nonlinear buckling analysis of shear deformable Mindlin plates. Two models of imperfections are introduced, one involving small uniform transverse loads and one involving distributed transverse loads corresponding to the number of half-waves indicated by the eigenvectors obtained from linear elastic buckling analysis. A simple numerical algorithm is presented to analyze the problems. Numerical examples with different geometries, loading and boundary conditions are used to demonstrate the accuracy of the formulations.

\section{Governing equations}

Figure 1 shows a geometrically nonlinear Mindlin plate. With the notation there, and with Greek indices varying from 1 to 2 and Roman indices from 1 to 3, the plate's governing equations can be written as

$$
M_{\alpha \beta, \beta}+Q_{\alpha}=0, \quad Q_{\alpha, \alpha}+\left(N_{\alpha \beta} w_{3, \beta}\right)_{, \alpha}+q=0 \quad N_{\alpha \beta, \beta}=0,
$$

Keywords: boundary element method, shear deformable plates, nonlinear buckling, imperfections. 

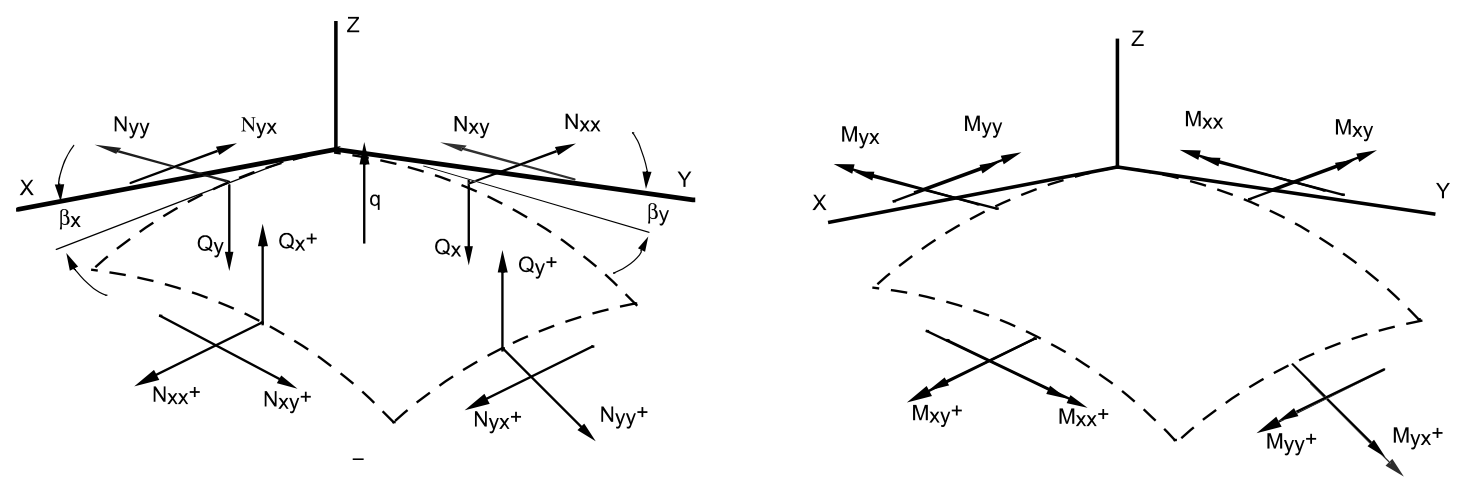

Figure 1. Stress resultant equilibrium in geometrically nonlinear plate element.

where $u_{\alpha}$ and $w_{3}$ are displacements in the $x_{\alpha}$ (in-plane) and $x_{3}$ (out-of-plane) directions; $w_{\alpha}$ are rotations in the $x_{\alpha}$ directions; $\delta$ is the Kronecker delta function; $Q_{\alpha}=C\left(w_{\alpha}+w_{3, \alpha}\right)$ and

$$
M_{\alpha \beta}=\frac{1-v}{2} D\left(w_{\alpha, \beta}+w_{\beta, \alpha}+\frac{2 v}{1-v} w_{\gamma, \gamma} \delta_{\alpha \beta}\right)+\frac{v}{\left(1-v^{2}\right) \lambda^{2}} q \delta_{\alpha \beta}
$$

are the stress resultants in plate bending problems, while $N_{\alpha \beta}=N_{\alpha \beta}^{\text {lin }}+N_{\alpha \beta}^{\text {nonlin }}$, with

$$
N_{\alpha \beta}^{\text {lin }}=\frac{1-v}{2} B\left(u_{\alpha, \beta}+u_{\beta, \alpha}+\frac{2 v}{1-v} u_{\gamma, \gamma} \delta_{\alpha \beta}\right), \quad N_{\alpha \beta}^{\text {nonlin }}=\frac{1-v}{2} B\left(w_{3, \beta} w_{3, \alpha}+\frac{2 v}{1-v} w_{3, \gamma} w_{3, \gamma} \delta_{\alpha \beta}\right),
$$

are the stress resultants for two-dimensional plane stress elasticity. The parameters are $B=E h /\left(1-v^{2}\right)$, the membrane stiffness; $D=E h^{3} /\left(12\left(1-v^{2}\right)\right)$, the bending stiffness of the plate; $q$, the transverse load; $C=D(1-v) \lambda^{2} / 2$, the shear stiffness; $E$, the modulus of elasticity; $\lambda=\sqrt{10} / h$, the shear factor; $h$, the thickness of the plate; $v$, the Poisson's ratio.

Extensive discussion on bending solutions of shear deformable plate theories can be found in [Wang et al. 2001].

\section{Boundary integral equations}

The boundary integral equation for the nonlinear buckling analysis of a plate bending can be written as

$$
\begin{array}{r}
C_{i j} w_{i}\left(x^{\prime}\right)+\int_{\Gamma} P_{i j}^{*}\left(x^{\prime}, x\right) w_{j}(x) d \Gamma=\int_{\Gamma} W_{i j}^{*}\left(x^{\prime}, x\right) p_{j}^{\operatorname{lin}}(x) d \Gamma+\int_{\Omega} W_{i j}^{*}\left(x^{\prime}, X\right) q(X) d \Omega(X) \\
+\int_{\Omega} W_{i 3}^{*}\left(x^{\prime}, X\right)\left(N_{\alpha \beta} w_{3, \chi}\right)_{, \alpha}(X) d \Omega(X) .
\end{array}
$$

The kernel solutions $P_{i j}$ and $W_{i j}$ can be found in [Aliabadi 2002]. The boundary integral equation for two-dimensional plane stress is expressed as

$$
C_{\theta \alpha}\left(x^{\prime}\right) u_{\alpha}\left(x^{\prime}\right)+\int_{\Gamma} T_{\theta \alpha}^{*}\left(x^{\prime}, x\right) u(x) d \Gamma=\int_{\Gamma} U_{\theta \alpha}^{*}\left(x^{\prime}, x\right) t^{\operatorname{lin}}(x) d \Gamma+\int_{\Omega} U_{\theta \alpha}^{*}\left(x^{\prime}, X\right) N_{\alpha \gamma, \gamma}^{\text {nonlin }}(X) d \Omega(X) .
$$


Using the divergence theorem, the domain integral on the right-hand side of (3) can be expressed as

$$
\begin{array}{r}
C_{\theta \alpha}\left(x^{\prime}\right) u_{\alpha}\left(x^{\prime}\right)+\int_{\Gamma} T_{\theta \alpha}^{*}\left(x^{\prime}, x\right) u(x) d \Gamma=\int_{\Gamma} U_{\theta \alpha}^{*}\left(x^{\prime}, x\right) t^{\operatorname{lin}}(x) d \Gamma+n_{\gamma}(x) \int_{\Gamma} U_{\theta \alpha}^{*}\left(x^{\prime}, x\right) N_{\alpha \gamma}^{\mathrm{nonlin}}(x) d \Gamma \\
-\int_{\Omega} U_{\theta \alpha, \gamma}^{*}\left(x^{\prime}, X\right) N_{\alpha \gamma}^{\mathrm{nonlin}}(X) d \Omega(X) .
\end{array}
$$

In a similar way, (4) can be simplified and written as

$$
\begin{aligned}
C_{\theta \alpha}\left(x^{\prime}\right) u_{\alpha}\left(x^{\prime}\right)+\int_{\Gamma} T_{\theta \alpha}^{*}\left(x^{\prime}, x\right) u(x) d \Gamma=\int_{\Gamma} U_{\theta \alpha}^{*}\left(x^{\prime}, x\right) t(x) d \Gamma & -n_{\gamma}(x) \int_{\Gamma} U_{\theta \alpha}^{*}\left(x^{\prime}, x\right) N_{\alpha \gamma}^{\mathrm{nonlin}}(x) d \Gamma \\
& +\int_{\Omega} U_{\theta \alpha}^{*}\left(x^{\prime}, X\right) N_{\alpha \gamma, \gamma}^{\mathrm{nonlin}}(X) d \Omega(X),
\end{aligned}
$$

where $t_{\alpha}=t_{\alpha}^{\text {lin }}+t_{\alpha}^{\text {nonlin }}$ and $t_{\alpha}^{\text {nonlin }}=N_{\alpha \gamma}^{\text {nonlin }} n_{\gamma}$. The fundamental solutions $U_{\theta \alpha}$ and $T_{\theta \alpha}$ are can be found in [Aliabadi 2002].

To calculate the nonlinear terms, two additional integral equations of the deflection $w_{3}$ and the in-plane stress resultants $N_{\alpha \beta}^{\text {lin }}$ at domain points are required:

$$
\begin{aligned}
w_{i}\left(X^{\prime}\right)+\int_{\Gamma} P_{i j}^{*}\left(X^{\prime}, x\right) w_{j}(x) d \Gamma=\int_{\Gamma} W_{i j}^{*}\left(X^{\prime}, x\right) p_{j}^{\operatorname{lin}}(x) d \Gamma & +\int_{\Omega} W_{i j}^{*}\left(X^{\prime}, X\right) q(X) d \Omega(X) \\
& +\int_{\Omega} W_{i 3}^{*}\left(X^{\prime}, X\right)\left(N_{\alpha \beta} w_{3, \chi}\right)_{, \alpha}(X) d \Omega(X),
\end{aligned}
$$

$$
\begin{aligned}
N_{\alpha \beta}^{\operatorname{lin}}\left(X^{\prime}\right)=\int_{\Gamma} U_{\Delta \alpha \beta}^{*}\left(X^{\prime}, x\right) t_{\Delta}(x) d \Gamma-\int_{\Gamma} T_{\Delta \alpha \beta}^{*}\left(X^{\prime}, x\right) u_{\Delta}(x) d \Gamma \\
\quad-n_{\gamma}(x) \int_{\Gamma} U_{\Delta \alpha \beta}^{*}\left(X^{\prime}, x\right) N_{\alpha \gamma}^{\text {nonlin }}(x) d \Gamma+\int_{\Omega} U_{\Delta \alpha \beta}^{*}\left(X^{\prime}, X\right) N_{\alpha \gamma, \gamma}^{\text {nonlin }}(X) d \Omega(X),
\end{aligned}
$$

where the fundamental solutions $U_{\Delta \alpha \beta}^{*}$ and $T_{\Delta \alpha \beta}^{*}$ can be found in [Aliabadi 2002].

The domain integrals appearing in (2), (5), (6), and (7) are evaluated by using the dual reciprocity technique as described in [Wen et al. 2000]. The particular solutions for plate bending and two-dimensional plane stress can also be found in the same reference.

\section{Evaluation of derivative terms}

The derivatives of deflection $w_{3, \gamma}$ on the boundary and in the domain can be approximated using a radial basis function $f(r)=\sqrt{c^{2}+r^{2}}$, where $r=\sqrt{\left(x_{1}-x_{1}^{m}\right)^{2}+\left(x_{2}-x_{2}^{m}\right)^{2}}$ :

$$
w_{3}\left(x_{1}+x_{2}\right)=\sum_{m=1}^{M+N} f(r)^{m} \Psi^{m},
$$

where $N$ and $M$ are respectively the number of selected points $x_{1}$ and $x_{2}$ on the boundary and in the domain. The $\Psi^{m}$ are coefficients which are determined by values at the selected points as follows:

$$
\Psi=F^{-1}\left\{w_{3}\right\}
$$


The derivatives of the deflection values may be expressed by

$$
w_{3, \gamma}\left(x_{1}+x_{2}\right)=f(r)_{, \gamma} F^{-1}\left\{w_{3}\right\} .
$$

The nonlinear terms $N_{\alpha \gamma, \gamma}^{\text {nonlin }}$ which appear in (5) and (7) can be evaluated in a similar way as well. Using this approach, there is no need to evaluate the derivatives of the transverse displacement $w_{3, \gamma}$ through the integral equations. The integral equations usually have complicated mathematical terms and may have singularities of higher order.

A relaxation procedure is used to improve the numerical results. As the nonlinear terms are calculated in each step $(k)$ of increments, the deflection $w_{3}$ can be modified as

$$
w_{3}^{k+1}=\frac{w_{3}^{k+1}+w_{3}^{k}}{2} .
$$

Note that the relaxation procedure shown in (11) works well for moderately low load levels. If higher load levels are applied, the use of (11) is not recommended.

\section{Imperfection models}

The initial imperfections of the transverse loads are introduced to trigger buckling modes. Figure 2 shows the two imperfection models used:

(i) uniform distribution of the transverse loads $q_{0}$ in the domain $\Omega$;

(ii) distributed transverse loads $q_{0}$ in the domain $\Omega$, corresponding to a number of half-waves indicated by the eigenvectors from the linear elastic buckling analysis [Purbolaksono and Aliabadi 2005a].

The first model only allows few nonlinear buckling problems to be accurately analyzed such as the geometries of square and circular models. The second model is generally recommended, since the imperfections can be modeled based on the eigenvectors that are related to the buckling modes. Hence, the second model may represent the initial imperfections that should be distributed in the domain.

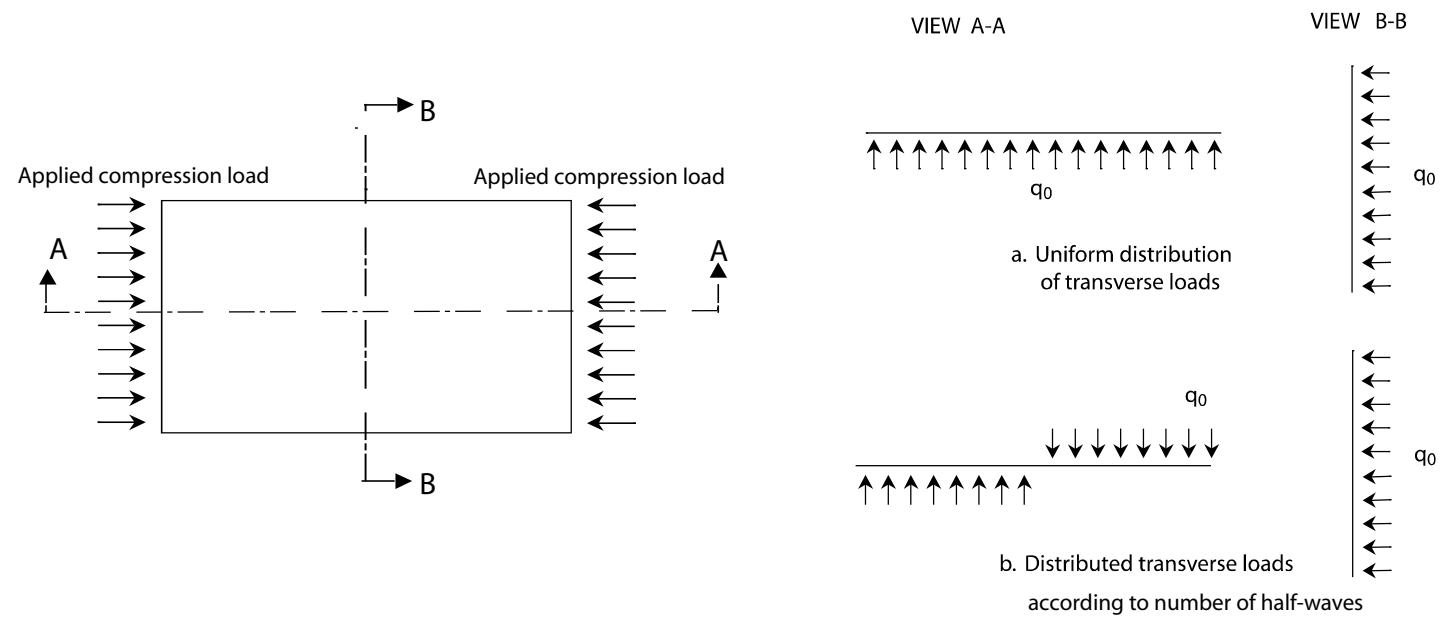

Figure 2. Initial imperfection models. 
The following equations are used to define the magnitudes of the load increment $\Delta \sigma$ and transverse loads $q_{0}$ throughout this work. The magnitudes are empirically maintained to be small enough. The relation between the load increment $\Delta \sigma$ and modulus of elasticity may be proposed as

$$
\frac{\Delta \sigma}{E} \approx X
$$

where $X$ is in the range of $10^{-7}$ to $5 \times 10^{-7}$. Next, the relation between the load increment $\Delta \sigma$ and transverse loads $q_{0}$ is proposed as

$$
q_{0}=\frac{\Delta \sigma_{h}}{5 b}
$$

where $b$ is the width or diameter of plates.

The transverse loads $q_{0}$ are used to introduce the initial imperfection loads in the plates according to the models shown in Figure 2.

\section{Numerical algorithms}

A simple numerical algorithm, requiring no iterations, is used to analyze nonlinear buckling problems. It can be summarized as follows:

Step 1: After introducing initial imperfection by uniform distribution $q_{0}$ or distributed transverse loads $q_{0}=q_{0}^{1}$ (see Figure 2) and a load increment $\Delta \sigma$, let the first step $k=1$ and the final step $k^{\text {final }}$ and initial values of $N_{\alpha \beta}^{\text {lin }}=0$ and $w_{, \alpha}=0$.

Step 2: Compute the coefficient matrices related to fundamental solutions. They can be stored in the core and used in each increment without any change.

Step 3: If $k \neq 1$ then $q_{0}^{k+1}=q_{0}^{k}+q_{0}^{1}$. Solve the linear system equation of the boundary integral equations to obtain boundary values. Then calculate the in-plane stress resultants $N_{\alpha \beta}^{\mathrm{lin}}$ and derivative of deflection $w_{, \alpha}$ in the domain.

Step 4: Apply the relaxation procedure given in (11). Then calculate the nonlinear terms $\left(N_{\alpha \gamma, \gamma}^{\text {nonlin }}\right)^{(k)}$ and $\left[\left(N_{\alpha \beta} w_{3, \beta}\right)_{, \alpha}\right]^{(k)}$ using approximation function as described in (6)-(8). The nonlinear terms will be used for the evaluation in the next step $k+1$.

Step 5: Calculate the nonlinear membrane traction $t_{\alpha}^{\text {nonlin }}$ on the boundary.

Step 6: Print results.

Step 7: If $k=k^{\text {final }}$, terminate; otherwise let step $k=k+1$ and go to Step 3 .

By introducing cumulative transverse loads $q_{0}^{k}$ at each step $k$, the equilibrium of (4) could be maintained. The transverse loads $q_{0}$ as the imperfection loads however might provide potential biasing of the results if they are arbitrarily defined.

\section{Numerical examples}

Several numerical examples with different geometries, loadings, and boundary conditions are presented to demonstrate the ability of the proposed method. Equations (12) and (13) are used to define the magnitudes of the load increment $\Delta \sigma$ and transverse loads $q_{0}$. 


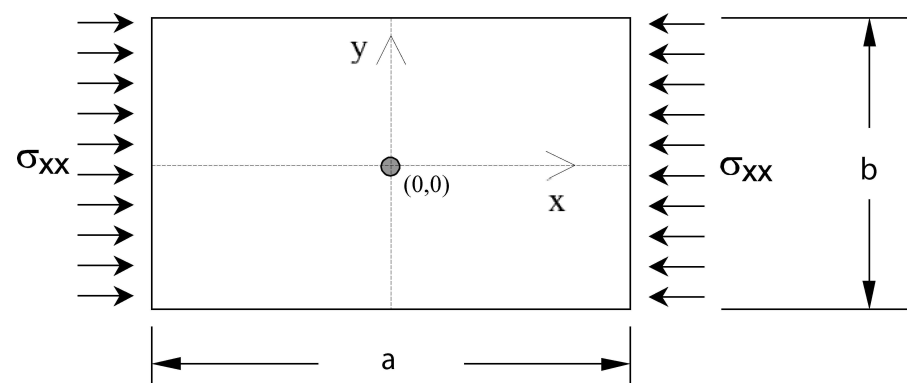

Figure 3. Nonlinear buckling model.

The nonlinear buckling model is shown in Figure 3. In the following examples, the normalized critical compression stress $K_{n l}$ is defined by

$$
K_{n l}=\frac{b^{2} h}{\pi^{2} D} \sigma
$$

where $\sigma$ is compression stress.

7.1. Convergence study of simply supported square plate subjected to uniaxial compression loads. In this example, a square plate subjected to compression loads at its ends as shown in Figure 3 is analyzed. Five different distributions of domain points are used for the dual reciprocity calculation. The initial imperfection is introduced by uniform transverse load $q_{0}=0.005$ units and in the case of $\Delta \sigma=4$ units. A convergence study of the simply supported square plate is performed and the normalized compression stresses $K_{n l}$ and the normalized deflection $Z\left(=w_{3} / h\right)$ are plotted in Figure 4. The results given in [Levy $1942]$ are also plotted in Figure 4. It can be seen that the convergence of the results can be achieved with 49 domain points. The normalized compression stress is in agreement with the critical value $K_{n l} \approx 4$ of the analytical result [Timoshenko and Gere 1961]. The BEM results are also in good agreement with Levy's solution [Levy 1942].

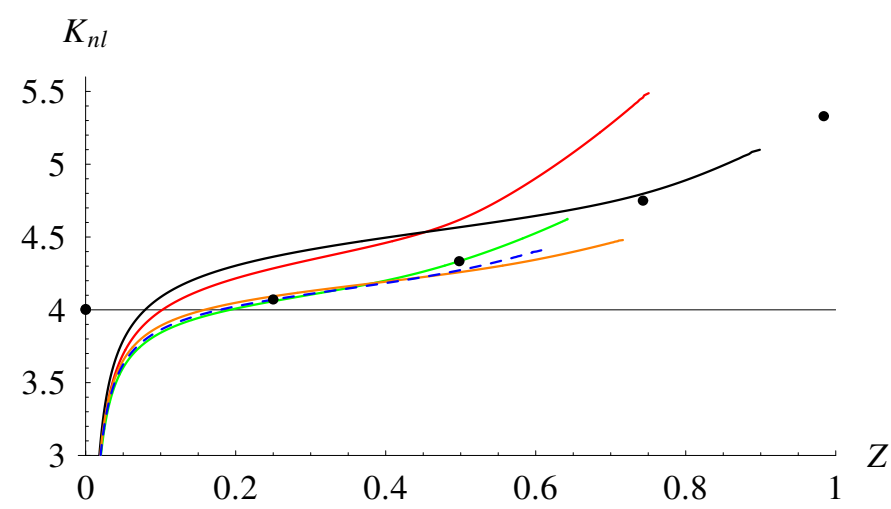

Figure 4. Normalized compression stresses $K_{n l}$ and deflection $Z$ for different numbers of domain points: from top to bottom at rightmost point, $5 \times 5,6 \times 6,7 \times 7$, and $8 \times 8$ (dashed curve). The black dots are values from [Levy 1942]. The thin horizontal line marks the critical value [Timoshenko and Gere 1961]. 

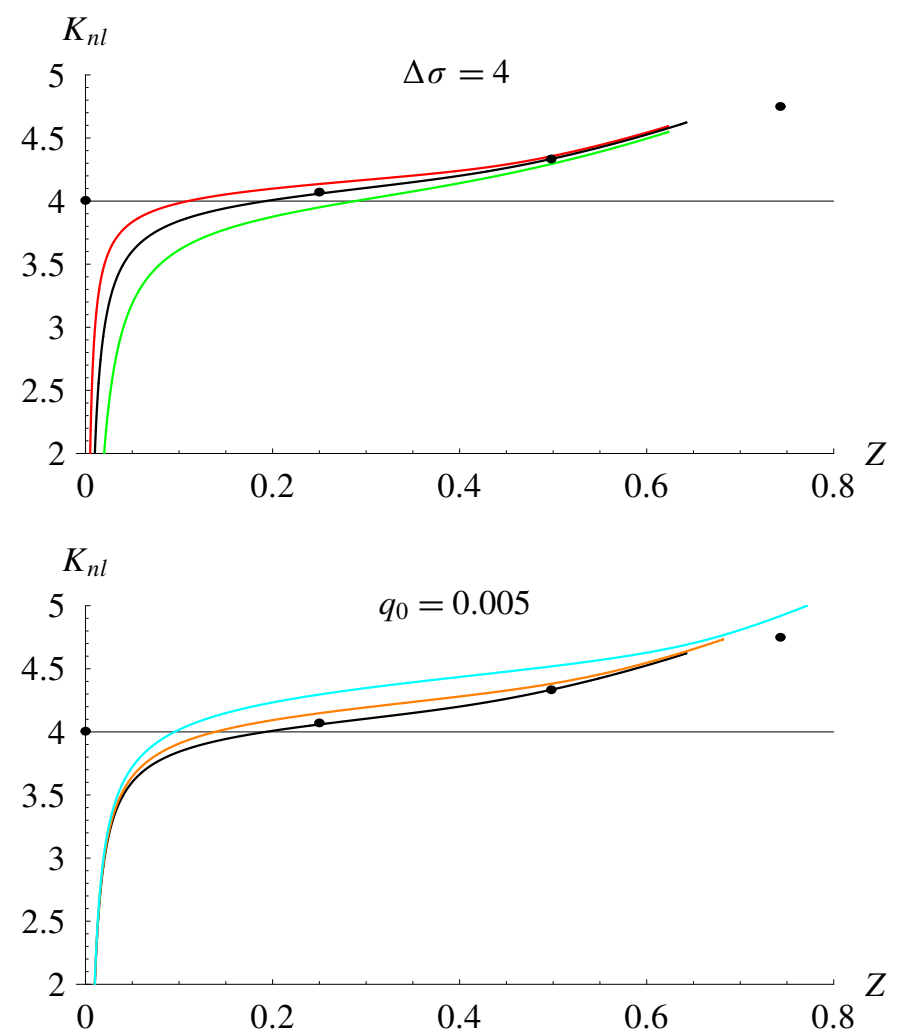

Figure 5. Normalized compression stresses $K_{n l}$ and deflection $Z$ for various transverse loads (top diagram; curves from top to bottom, $q_{0}=0.0025,0.005,0.01$ ) and for various increments of the compression load (bottom diagram: curves from from top to bottom, $\Delta \sigma=16,8,4)$. Black dots and horizontal line as in Figure 4.

7.2. Simply supported square plate subjected to uniaxial compression loads with different initial imperfections and increments of load. In this example, a simply supported square plate subjected to uniaxial compression load is analyzed with different imperfections and increments of the load. BEM meshing with 20 quadratic boundary elements and 49 domain points are used. The normalized compression stresses $K_{n l}$ and deflection $Z$ for different initial imperfections and in the case of $\Delta \sigma=4$ unit of compression loads are plotted in Figure 5, top.

The normalized compression stresses $K_{n l}$ and deflection $Z$ for different increment of compression loads and in the case of $q_{0}=0.005$ units of uniform transverse loads are plotted in Figure 5, bottom. It can be seen that the bigger value of initial imperfection provides a lower critical buckling load. The same graph also shows that the bigger value of compression load increment provides a bigger critical buckling load.

7.3. Circular and square plates subjected to a uniform normal compression loads. We performed the nonlinear buckling analysis of circular and square plates subjected to uniform normal compression loads (Figure 6). Two boundary conditions, simply supported and clamped, are applied. The BEM meshes used had 16 quadratic boundary elements and 33 domain points for the circular plate, and 20 quadratic 

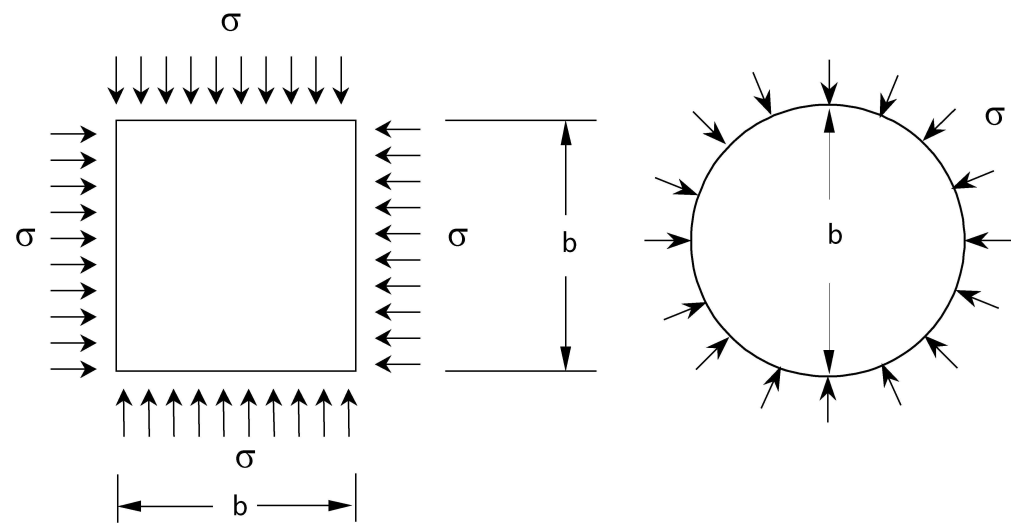

Figure 6. Circular and square plates subjected to uniform normal compression loads.

boundary elements and 25 domain points for the square plate. The compression load increments were chosen as $\Delta \sigma=4$ units and the initial imperfection as $q_{0}=0.005$ units. Figure 7 shows the normalized compression stresses $K_{n l}$ and the normalized deflection $Z$ together the with critical value of each model for linear elastic buckling analysis [Timoshenko and Gere 1961]. The results are seen to be agreement with the critical values.

7.4. Analysis of two imperfection models on simply supported rectangular plates. In this example, two imperfection models, namely uniform distribution and distributed transverse loads, are evaluated. A simply supported rectangular plate as shown in Figure 3 is used to investigate the proposed imperfection models. The origin is at the center of the plate. In the case of uniform distribution, the increments of compression loads as $\Delta \sigma=4$ units and initial imperfection as $q_{0}=0.005$ units are applied.

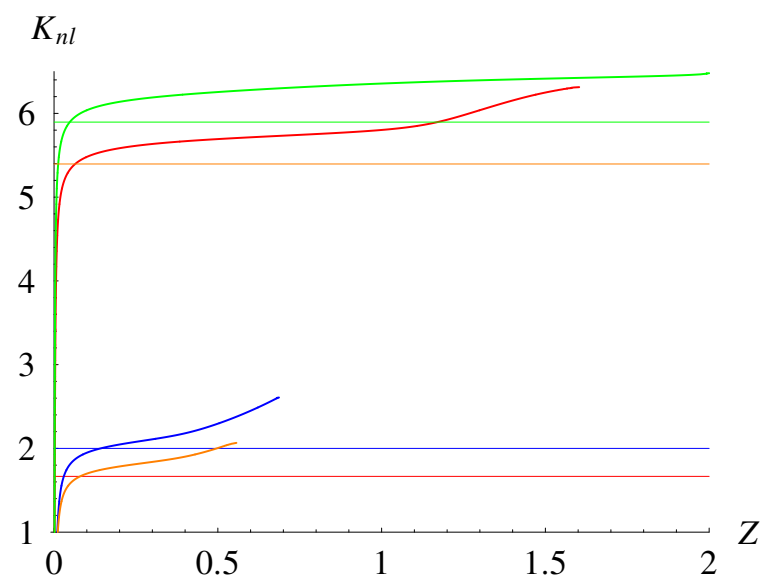

Figure 7. Normalized stresses $K_{n l}$ of circular and square plates subjected to uniform normal compression loads. Curves from top to bottom correspond to clamped circle, clamped square, simply supported square, and simply supported circle configuration. The horizontal lines show the critical values in the same order. 


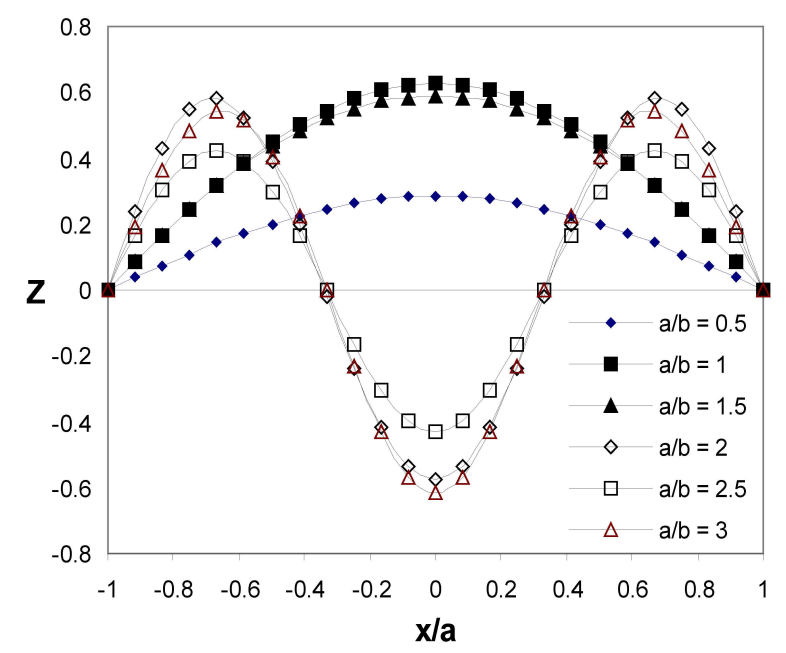

Figure 8. Half-wave modes for rectangular plates with different aspect ratio $a / b$ due to uniform imperfections.

The normalized deflections $Z$ for the points along $x$-axis due to uniform imperfections are plotted in Figure 8 . It can be seen that the plates will buckle in odd number of half-waves for different aspect ratios $a / b$.

For the distributed transverse load model, imperfections are introduced according to the buckling modes defined by the eigenvectors from linear elastic buckling analysis for the corresponding geometry. For the rectangular plate, the distribution of imperfections is shown in Figure 9.

The estimated normalized compression stresses $K_{n l}$ for different aspect ratio of the plates are plotted in Figure 10. It can be seen that the uniform imperfection of transverse loads provides inaccurate results with the increasing of aspect ratios. Moreover, for aspect ratios $a / b$ between 1.4 and 2.5, the buckling deformations of the plate do not form two half-wave modes as expected. The results obtained with the distributed loads according to the buckling modes are in good agreements with the published results.

7.5. Nonlinear buckling analysis of rectangular plates with different boundary conditions. We next turn to a nonlinear buckling analysis of rectangular plates as shown in Figure 3 subjected to a uniform

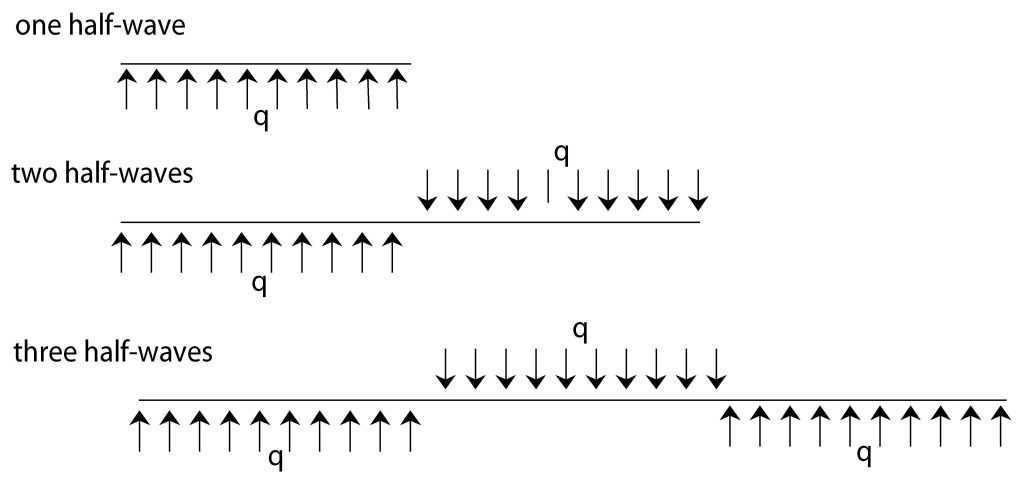

Figure 9. Simplified imperfections for rectangular plates. 


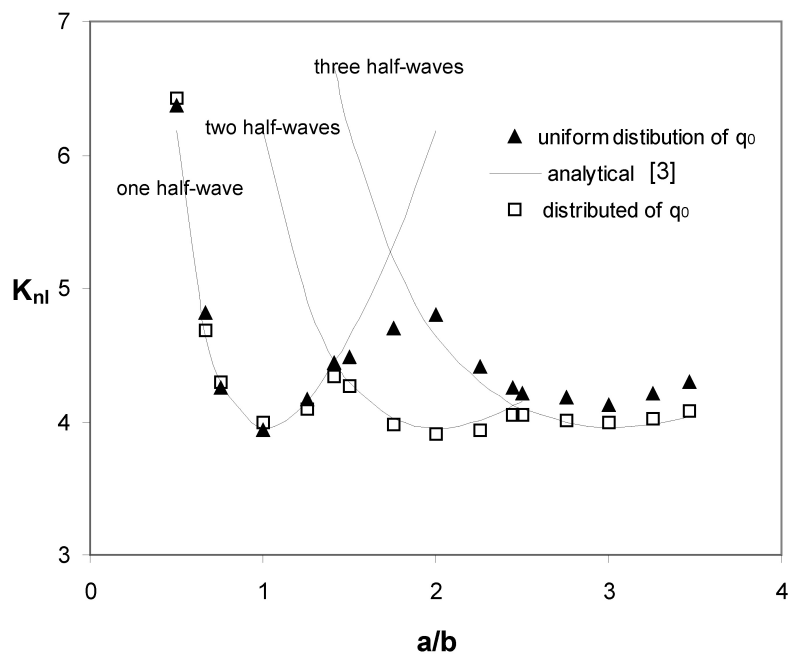

Figure 10. The normalized compression stresses $K_{n l}$ for different aspect ratio of the simply supported rectangular plates.

normal compression loads. Three boundary conditions are applied: all sides clamped (cccc), two opposite loaded side clamped and two others simply supported (cscs), and three sides simply supported and one unloaded side free (sssf). The deformations for rectangular plates with the these boundary conditions are shown in Figure 11.

The normalized compression stresses $K_{n l}$ and the normalized deflection $Z$ together with the critical value of each the three models above are plotted in Figure 12.

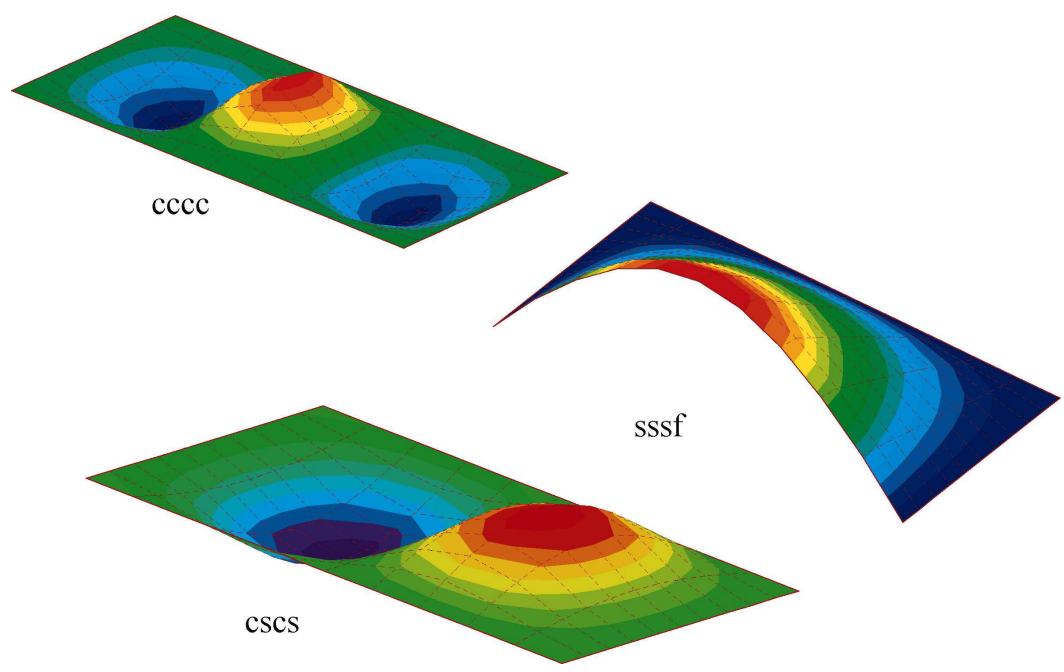

Figure 11. Nonlinear buckling deformations for rectangular plates with different boundary conditions. See text immediately above for abbreviations. 

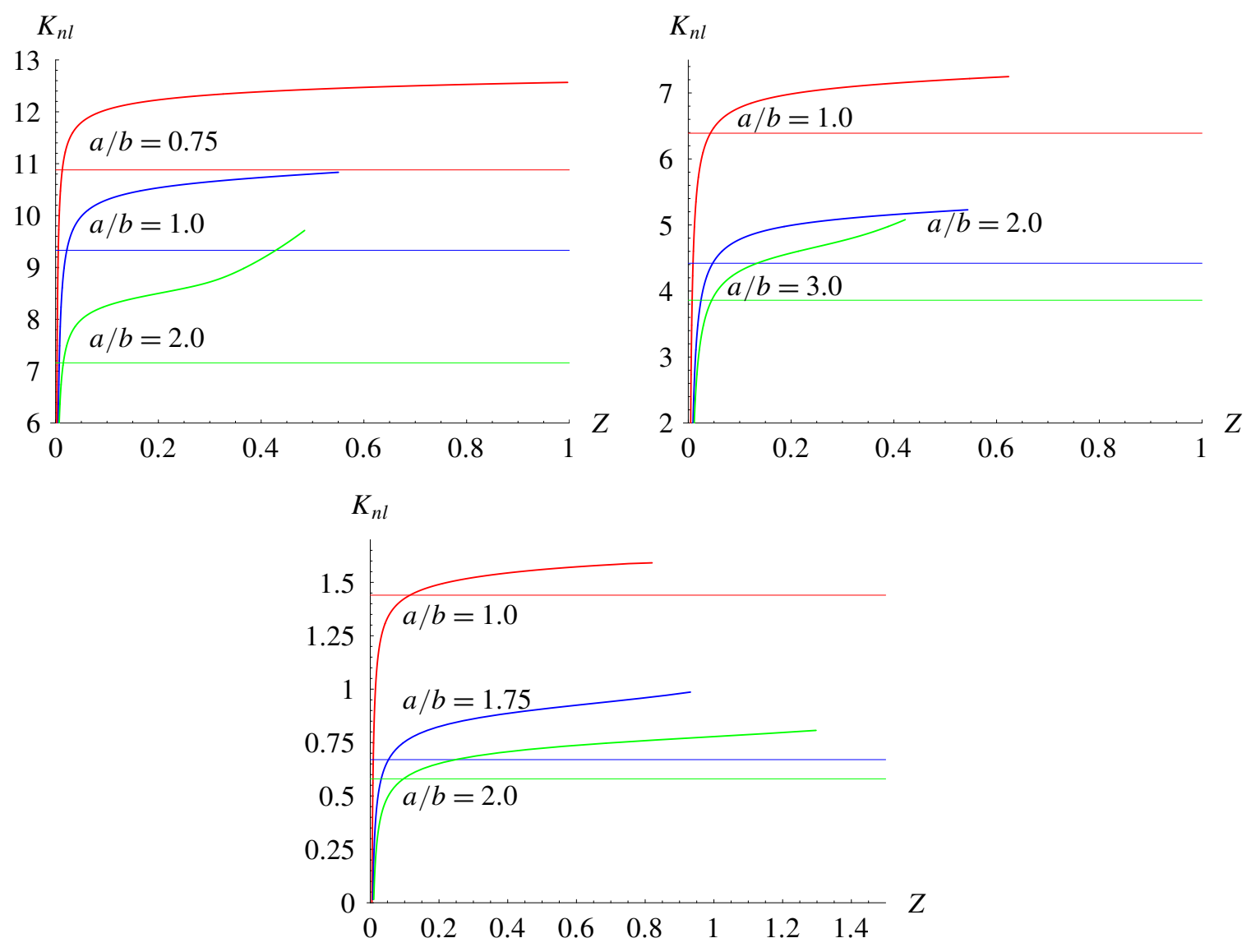

Figure 12. Normalized compression stresses $K_{n l}$ of rectangular plates, for different values of $a / b$ (given next to the curves to which they apply). Top left: all sides clamped. Top right: two opposite loaded sides clamped and two others simply supported. Bottom: three sides simply supported and one unloaded side free. The critical values (horizontal lines) are taken from [Purbolaksono and Aliabadi 2005a].

\section{Conclusions}

The BEM results obtained by using imperfections of the distributed transverse loads corresponding to the expected buckling modes were in good agreement with the published results and the theoretical critical buckling strengths. The proposed equations for defining the magnitudes of the load increment $\Delta \sigma$ and transverse loads $q_{0}$ reasonably also demonstrated the accuracy of the results for the analyses.

\section{Acknowledgments}

The authors thank Queen Mary and Westfield Research Council, University of London, United Kingdom for financial support during the completion of this work. 


\section{References}

[Aliabadi 2002] M. H. Aliabadi, The boundary element method, applications in solids and structures, vol. 2, Wiley, Chichester, 2002.

[Bao et al. 1997] G. Bao, W. Jiang, and J. C. Roberts, "Analytic and finite element solutions for bending and buckling of orthotropic rectangular plates", Int. J. Solids Struct. 34:14 (1997), 1797-1822.

[Brush and Almroth 1975] D. O. Brush and B. Almroth, Buckling of bars, plates, and shells, McGraw-Hill, New York, 1975.

[He and Qin 1993] X. Q. He and Q. H. Qin, "Nonlinear analysis of Reissner's plate by the variational approaches and boundary element methods", Appl. Math. Model. 17:3 (1993), 149-155.

[Kamiya et al. 1984] N. Kamiya, Y. Sawaki, and Y. Nakamura, "Postbuckling analysis by the boundary element method", Eng. Anal. 1:3 (1984), 40-44.

[Lei et al. 1990] X. Y. Lei, M. K. Huang, and X. X. Wang, "Geometrically nonlinear analysis of a Reissner type plate by the boundary element method", Comput. Struct. 37:6 (1990), 911-916.

[Levy 1942] S. Levy, Bending of rectangular plates with large deflections, National Advisory Committee for Aeronautics, 1942, Available at http://naca.central.cranfield.ac.uk/reports/1942/naca-report-737.pdf. TN-737.

[Liu 1987] Y. Liu, "Elastic stability analysis of thin plate by the boundary element method - new formulation", Eng. Anal. 4:3 (1987), 160-164.

[Liu 2001] F. L. Liu, "Differential quadrature element method for buckling analysis of rectangular Mindlin plates having discontinuities", Int. J. Solids Struct. 38:14 (2001), 2305-2321.

[Manolis et al. 1986] G. D. Manolis, D. E. Besko, and M. F. Pineros, "Beam and plate stability by boundary elements", Comput. Struct. 22:6 (1986), 917-923.

[Marczak and de Barcellos 1998] R. J. Marczak and C. S. de Barcellos, "A boundary element formulation for linear and nonlinear bending of plates", in Proc. Fourth World Congress of Computational Mechanics, IACM, 1998.

[Purbolaksono and Aliabadi 2005a] J. Purbolaksono and M. H. Aliabadi, "Buckling analysis of shear deformable plates by boundary element method", Int. J. Numer. Methods Eng. 62:4 (2005), 537-563.

[Purbolaksono and Aliabadi 2005b] J. Purbolaksono and M. H. Aliabadi, "Dual boundary element method for instability analysis of cracked plates", Comput. Model. Eng. Sci. 8:1 (2005), 73-90.

[Qin and Huang 1990] Q. Qin and Y. Huang, "BEM of postbuckling analysis of thin plates", Appl. Math. Model. 14:10 (1990), 544-548.

[Syngellakis 1998] S. Syngellakis, Stability plate bending analysis with boundary elements, edited by M. H. Aliabadi, Computational Mechanics Publications, Southampton and Boston, 1998.

[Tanaka et al. 1999] M. Tanaka, T. Matsumoto, and Z. Zheng, "Application of the boundary-domain element method to the pre/post-buckling problem of von Karman plates”, Eng. Anal. Bound. Elem. 23:5-6 (1999), 399-404.

[Timoshenko and Gere 1961] S. P. Timoshenko and J. M. Gere, Theory of elastic stability, 2nd ed., McGraw-Hill, New York, 1961.

[Walker 1984] A. C. Walker, "A brief review of plate buckling research", in Behaviour of thin-walled structures, edited by J. Rhodes and J. Spence, Elsevier, London, 1984.

[Wang et al. 2001] C. M. Wang, G. T. Lim, J. N. Reddy, and K. H. Lee, "Relationships between bending solutions of Reissner and Mindlin plate theories", Eng. Struct. 23:7 (2001), 838-849.

[Wen et al. 2000] P. H. Wen, M. H. Aliabadi, and A. Young, "Application of dual reciprocity method to plates and shells", Eng. Anal. Bound. Elem. 24:7-8 (2000), 583-590.

Received 12 Dec 2008. Revised 23 Jun 2009. Accepted 4 Jul 2009.

Judha Purbolaksono: judha@uniten.edu.my

Department of Mechanical Engineering, Universiti Tenaga Nasional, Km 7 Jalan Kajang-Puchong, Kajang 43009, Selangor, Malaysia

M. H. (FERRI) AliABADI: m.h.aliabadi@imperial.ac.uk

Department of Aeronautics, Faculty of Engineering, Imperial College London, Prince Consort Road, London SW7 2BY,

United Kingdom 